\title{
AMMISSIONE DI STRANIERI SUL TERRITORIO NAZIONALE ATTRAVERSO 'CORRIDOI UMANITARI' CON SPONSOR PRIVATI
}

\author{
CAROLA RICCI (*)
}

Nota presentata dal m.e. Fausto Pocar

(Adunanza del 4 ottobre 2018)

\begin{abstract}
SunTO. - Lo scopo della ricerca che si presenta è comprendere quanto una iniziativa privata di sponsorizzazione di ingressi legali alternativi di migranti bisognosi di protezione, inaugurata di recente in Italia e denominata "corridoi umanitari", possa diventare in futuro un modello uniforme diffuso in altri Stati europei e se, a tale scopo, debba essere diversamente modulata. Infatti, tale buona pratica ad oggi di natura eccezionale, ha permesso un ingresso non rischioso, per 'via aerea', nel nostro Paese di soggetti vulnerabili provenienti in via principale dal Libano, ma anche dall'Eritrea. I beneficiari individuati, una volta sottoposti ai dovuti controlli di sicurezza, sono stati accolti da comunità e famiglie sponsor che si sono rese disponibili ad aiutarli nella cruciale iniziale fase di integrazione. Tale prassi potrebbe essere estesa in altri Stati europei perché fondata non tanto e non solo sull'art. 25 del c.d. Codice Visti (di recente purtroppo svuotato di contenuto obbligatorio dalla Corte di giustizia UE e indicato finora come sua base giuridica), ma soprattutto su diverse regole di diritto internazionale (sia generale sia convenzionale) da cui discende il dovere posto in capo agli Stati e alla società civile di adoperarsi, secondo un modello di 'alleanza pubblico-privato', al fine di trovare soluzioni a sfide e potenzialità poste dal fenomeno delle migrazioni internazionali di soggetti vulnerabili, come indicato nella Dichiarazione di New York del 2016.
\end{abstract}

$* * *$

ABSTRACT. - The scope of the present research is to understand to what extent a recent and fruitful private initiative sponsoring a safe alternative legal pathway 'par avion' recently spread from Italy and called "humanitarian corridors", may in a future become

(") Professore associato di Diritto internazionale nell'Università di Pavia, Italy. E-mail: carola.ricci@unipv.it 
a general and uniform alternative model for other European Union States. Such a best practice, which represents currently an exceptional route for vulnerable migrants mostly from Lebanon and Eritrea to enter the country without harm after a security screening and to be materially supported by the same sponsors in the crucial initial phase of integration, could potentially be extended to other EU States. Its legal basis should not be restricted to Article 25 of the Visa Code (recently interpreted by the EU Court of Justice as posing no obligation on Member States to grant humanitarian visa). There already exist clear obligations to grant humanitarian assistance to vulnerable people at risk stemming out from international law (both general and conventional) that do constitute the adequate legal basis both for States and civil society, to act in a "multi-stakeholder alliance" in order to find solutions to the challenges and opportunities deriving from international migration, as indicated in the 2016 New York Declaration.

\section{SPUNTI INTRODUTTIVI}

Lo studio che è presentato all'Adunanza odierna sul tema delle vie legali alternative all'ingresso di migranti in stato di necessità si inserisce tra le tematiche affrontate dal gruppo di ricerca interdisciplinare impegnato nel Tema Strategico dell'Ateneo (TSA) di Pavia dedicato a sviluppare un nuovo modello interdisciplinare di governance per il fenomeno migratorio, denominato "MIGRAT.IN.G. (MIGRATions: towards an INterdisciplinary Governance)", un team che coinvolge quasi cento studiosi di scienze biomediche, sociali e umane.

Sono passati cinque anni da quel terribile 3 ottobre 2013, quando 368 persone, per lo più donne e bambini eritrei, persero la vita in un naufragio al largo di Lampedusa. Una tragedia che si è ripetuta dolorosamente più volte negli ultimi anni. Si calcola che dal 1990 ad oggi oltre 37 mila persone siano morte in mare, nel tentativo di raggiungere l'Europa. Nonostante il netto calo degli arrivi dei migranti attraverso il Mediterraneo, nel 2018 è aumentata la percentuale delle vittime in mare, a motivo di viaggi sempre più pericolosi.

L'obbligo di soccorso di natanti in difficoltà e il divieto dei respingimenti di massa sono fissati dal diritto internazionale, non solo convenzionale (convenzione di Montego Bay del 1984, convenzione di Ginevra sullo status dei rifugiati del 1951 e il suo protocollo del 1967, Convenzione di New York del 1989 sui diritti del fanciullo) ma anche consuetudinario (non refoulement, divieto di tortura e trattamenti inumani e degradanti...), e sono confermati dalla giurisprudenza di corti interne e internazionali (in particolare della Corte di Strasburgo).

Ciò premesso, però, la citata convenzione di Ginevra sullo status dei rifugiati viene interpretata nel senso di prevedere un obbligo di tutela in capo allo Stato parte solo quando gli sfollati giungano sul suo territorio o comunque 
quando questo eserciti sui rifugiati la propria giurisdizione, definita come "controllo diretto ed effettivo". Non è cioè loro estesa la protezione quando essi si trovino sotto la giurisdizione di uno Stato terzo, non parte della convenzione.

Il sistema che ne deriva costringe tutti coloro che fuggono dal loro paese perché non hanno più alternative per la sopravvivenza a tentare pericolosi viaggi della speranza che li portino anche via mare a finire in aree controllate dallo Stato a cui vorrebbero chiedere protezione. Si tratta sia di perseguitati per motivi di razza, religione, convinzioni politiche e appartenenza a un determinato gruppo sociale (secondo la definizione di rifugiato contenuta nella convenzione di Ginevra '51), sia di quanti comunque fuggono da un conflitto armato (non tecnicamente 'rifugiati') o, ancora, da condizioni di vita insopportabili.

Il drammatico bilancio del numero crescente di persone che perdono la vita in mare cercando asilo e protezione internazionale rende sempre più urgente favorire vie legali alternative di accesso a Stati sicuri e politiche di integrazione efficaci che sostengano buone pratiche, come il progetto dei corridoi umanitari, oggetto della breve presentazione odierna.

Totalmente finanziato dalle comunità religiose promotrici (Comunità di Sant'Egidio insieme a Chiese protestanti italiane, Tavola valdese e CEI), finora ha prodotto risultati molto positivi ed è diventato già un modello virtuoso, avendo ottenuto l'adesione non solo del governo italiano, ma anche di altri Paesi europei come Francia e Belgio. Nonostante in termini assoluti le cifre sembrino ridotte, le stesse comunque si attestano su flussi di ingressi che superano quelle di diversi Stati membri dell'Unione europea. Si tratta per il momento di duemila beneficiari provenienti da campi profughi in Libano (per lo più siriani) e in Etiopia (profughi del Corno d'Africa), duemila persone giunte in sicurezza in Italia, con un volo di linea, e accolte sul territorio dove hanno ricevuto non solo ospitalità dalle comunità individuate dalle diverse associazioni in centri urbani non periferici, sparsi su tutto il territorio, ma anche gli strumenti (inclusi corsi di lingua e professionalizzanti) per integrarsi al meglio nel tessuto sociale e lavorativo italiano e/o cure mediche improcrastinabili. Questo progetto pilota sta offrendo a persone in stato di particolare bisogno e vulnerabilità forme innovative di accoglienza e di protezione internazionale, anche in casi in cui non siano state individuate prima facie meritevoli di riconoscimento dello status di rifugiati dall'UNHCR, ma si trovino comunque in specifiche situazioni individuali di vulnerabilità, a causa delle quali non avrebbero alcuna possibilità di raggiungere l'Europa in sicurezza e anzi facilmente cadrebbero vittime del traffico o della tratta di esseri umani. 


\section{L'IMPEGNO A CERCARE VIE D'INGRESSO LEGALI ALTERNATIVE: IL PRIVATE SPONSORSHIP MODEL SBARCA IN ITALIA}

Nella Dichiarazione di New York del settembre 2016 su rifugiati e migranti, gli Stati membri delle Nazioni Unite si sono impegnati a realizzare diversi obiettivi tra cui potenziare le vie legali alternative di accesso al territorio di Stati «sicuri» per i rifugiati in modo coordinato insieme alla società civile e al settore privato.

Tra le buone prassi adottate per consentire alle persone bisognose di protezione internazionale di raggiungere il Paese di destinazione in condizioni di piena legalità e sicurezza, il già consolidato modello canadese di private sponsorship ha attecchito anche in Italia, rendendo possibile nell'arco di due anni l'ingresso sul territorio di 1.000 profughi siriani in prevalenza provenienti dal Libano (di cui circa 700 sono già giunti) ai quali, nel corso del 2018, si sono aggiunti nuovi arrivi anche dai campi profughi dell'Etiopia (che raccolgono esuli provenienti dagli Stati del Corno d'Africa, Eritrea, Somalia, Sud Sudan) e del Marocco.

Questi "corridoi umanitari" sono stati aperti grazie a tre protocolli di intesa ibridi. I primi due sono stati stipulati per i beneficiari siriani provenienti principalmente dal Libano (e dal Corno d'Africa), il 15 dicembre 2015 e il 12 gennaio 2017, tra i Ministeri degli Affari Esteri e dell'Interno, dal lato istituzionale, e, rispettivamente, dal lato degli sponsor privati, la Comunità di Sant'Egidio, la Tavola Valdese, la Federazione delle Chiese evangeliche (parti del primo accordo) e la CEI (che, grazie a Caritas e Fondazione Migrantes, partecipa al secondo accordo insieme ancora alla Comunità di Sant'Egidio). Il più recente accordo è stato firmato il 7 novembre 2017 dalle stesse parti contraenti del primo protocollo di cui ricalca i contenuti in modo speculare ma è esteso ai profughi provenienti dal Marocco.

I protocolli prevedono il rilascio di un visto di ingresso per motivi umanitari ai sensi dell'art. 25 del regolamento (CE) n. $810 / 2009$, c.d. codice dei visti. Pare utile ricordare che, come pur noto, quest'ultima disposizione, in deroga al principio dell'adempimento delle condizioni di ingresso di cui all'art. 5 par. 1 lettere $a, c, d$, $e$ del Codice frontiere Schengen, riconosce a ciascuno Stato Membro la facoltà di rilasciare un visto, laddove lo ritenga necessario, «per motivi umanitari o di interesse nazionale o in virtù di obblighi internazionali», con una "validità territoriale limitata" (VTL) al proprio Stato per non più di 90 giorni (su un periodo totale di 180 giorni), a 
meno che - in via eccezionale - altri Stati membri abbiano acconsentito a estenderlo al proprio territorio.

Si tratta di comprendere quanto tale peculiare procedura di ingresso protetto (indicata con l'acronimo 'PEP'), ad oggi via d'ingresso legale di natura eccezionale, possa diventare in futuro prassi (se non regola) diffusa in altri Stati europei e se, a tale scopo, debba essere diversamente modulata o modificata. In particolare, in seguito alla sentenza X e X c. Stato Belga, decisa dalla Corte UE il 7 marzo dell'anno scorso (in causa C-638/16 PPU), non sembra che si possa mantenere formalmente la stessa base giuridica richiamata espressamente nelle intese in esame per i motivi che si intende chiarire.

\section{SCOPI}

Attualmente i protocolli d'intesa specificano, in modo quasi speculare (presentandosi con identica numerazione e pressoché identici contenuti), che gli scopi principali sono:

a) non solo l'arrivo in Italia di «potenziali beneficiari di protezione internazionale, in specie i soggetti più vulnerabili» in modo legale $e$ sicuro dallo Stato terzo ove si trovino e dove abbiano trovato rifugio, a spese delle comunità religiose richiamate (art. 2), ma anche

b) l'accoglienza in una fase iniziale, sempre ad opera della rete di volontari disseminati sul territorio nazionale che fanno capo alle stesse comunità, con modalità che possano garantire l'effettivo sostegno nel processo di «inserimento socioculturale per un congruo periodo di tempo», un anno (art. 4, secondo par.), oltre che

c) la «stabilizzazione in Italia» dei beneficiari, assicurando anche l'assistenza legale necessaria a richiedere protezione internazionale nel nostro Paese una volta che vi siano giunti - e solo nel nostro Paese, per buona pace degli altri Stati membri sono evitati movimenti secondari (art. 4 lett. $d$ della prima intesa e punto $11 c$ delle Considerazioni della seconda e terza intesa).

\section{I BENEFICIARI}

Per quanto concerne l'individuazione dei beneficiari, sono le ONG stesse a predisporre i dossier individuali in piena autonomia, acquisendo 
le informazioni necessarie direttamente sul terreno, nei campi profughi, eventualmente con il supporto dell'UNHCR. In questa fase viene loro rimessa una notevole discrezionalità nel vagliare i potenziali beneficiari e predisporne una lista di selezionati sulla base dei criteri individuati nei protocolli e connaturati allo stesso modello di sponsorship (art. 3).

Beneficiari potranno essere dunque:

i) non solo quelle «persone riconosciute meritevoli dall'UNCHR, almeno prima facie, del riconoscimento dello status di rifugiato» ai sensi della Convenzione di Ginevra del 1951 (e del Protocollo del 1967), ma anche

ii) i potenziali richiedenti protezione comunque in condizioni di vulnerabilità, anche se con alcune notevoli differenze tra i due accordi: nel primo protocollo, si è prevista l'estensione sia alle persone per le quali «esista il fondato motivo di ritenere che, nel caso di rientro nel paese di origine, possano subire un danno grave anche come conseguenza di una situazione di conflitto armato, di violenza endemica o di violazione sistematica dei diritti umani» (art. 3 lett. $b$ ); sia a tutti i migranti che si trovino «in una particolare condizione di vulnerabilità, per la loro situazione personale, l'età o le condizioni di salute» tra cui, a titolo esemplificativo, si annoverano le «donne sole con bambini, donne sole vittime di tratta, minori non accompagnati, anziani», disabili, vittime di traumi o in gravi condizioni di salute (art. 3 lett. c). Nel secondo e terzo protocollo, invece, l'estensione riguarda solamente le persone che si trovino in gravi e consistenti condizioni di «vulnerabilità determinata dalla loro situazione personale, dall'età e dalle condizioni di salute», senza peraltro che sia data indicazione di alcuna categoria specifica, neppure a titolo esemplificativo (art. 3 lett. $b$ ).

Nella stessa disposizione presente in tutti i testi si specifica però che nell'individuazione dei beneficiari si terrà conto «in forma complementare e non sostitutiva» anche di ulteriori fattori, includendo nel programma:

iii) quanti già possono usufruire in Italia di una «disponibilità all'accoglienza da parte di singoli, chiese o associazioni che provvedano inizialmente alla loro ospitalità» (art. 3 lett. d) e/o hanno «reti familiari $o$ sociali stabili in Italia e per questa ragione hanno dichiarato di volersi stabilire ed integrare nel nostro paese» (art. 3 lett. e), al fine espresso di «facilitare l'individuazione di percorsi di integrazione ed escludere o limitare eventuali movimenti secondari volontari» (art. 3 ultimo capoverso). 
La selezione quindi è seguita dalla firma di un accordo tra la comunità promotrice e i potenziali beneficiari con cui la prima si obbliga a garantire il viaggio, l'accoglienza e l'avviamento all'autonomia sociale ed economica oltre che ad avviare le pratiche per l'ottenimento della protezione internazionale in Italia, mentre i secondi si impegnano a integrarsi e accettare di essere accompagnati in questo processo di integrazione e stabilizzazione in Italia. Un progetto condiviso è dunque alla base di questo peculiare modello di sponsorizzazione privata.

Quindi seguono i processi di identificazione e i controlli di sicurezza preventivi svolti dal ministero dell'Interno e degli Esteri che ricevono i dossier dai consolati nei paesi di rifugio. Infine, solo dopo i controlli richiesti e in caso non ci siano motivi ostativi, viene rilasciato un VTL, sulla base appunto dell'art. 25 del codice visti.

\section{LA COMPATIBILITÀ DEL MODELLO CON LA BASE GIURIDICA INDICATA NELL'ART. 25 DEL CODICE DEI VISTI DOPO LA SENTENZA X E X C. BELGIO}

Specificati gli scopi e i beneficiari dei protocolli occorre brevemente soffermarsi sulla loro base giuridica, l'art. 25 del codice visti, perché, nella già citata sentenza della Corte di giustizia, viene esclusa l'operatività di tale disposizione $\mathrm{a}$ un caso che potenzialmente ricadrebbe nelle stesse intese italiane in commento, intese che proprio su quella si proclamano fondate.

A Lussemburgo si è statuito che uno Stato membro, nel caso di specie il Belgio, può rifiutare (anche senza darne motivazione) una domanda di visto VTL presentata alla rappresentanza consolare belga in Libano sulla base dell'art. 25 del codice visti da una famiglia di profughi siriani (inclusi tre figli minori), cristiani ortodossi sottoposti a persecuzione ad Aleppo, quando dalla domanda si tragga la loro intenzione di richiedere protezione internazionale una volta arrivati in Belgio.

Una simile domanda, infatti, secondo la Corte, sottintenderebbe la volontà di permanere sul territorio ben oltre i 90 giorni prescritti dal codice dei visti, con la conseguente inapplicabilità non solo del codice stesso ma anche del diritto dell'Unione europea oltre che degli standard di protezione posti dalla Carta dei diritti fondamentali, in particolare dagli artt. 4 e 18 che riconoscono rispettivamente il divieto di tortura e trattamenti inumani e degradanti e il diritto di asilo. 
La Corte arriva a tale (assai criticabile) conclusione sulla base della constatazione che:

i) l'espressa intenzione di richiedere protezione internazionale contenuta formalmente in una domanda VTL è causa di non applicazione del codice visti (e non invece possibile motivo di rifiuto ex art. 32 par. 1 lett. $b$ del codice stesso): avendo un oggetto differente da quello di un visto di breve durata, deve essere qualificato come visto di lunga durata (parr. 43, 46-47);

ii) la competenza in materia di definizione dei criteri uniformi per il rilascio di visti o titoli di soggiorno di lunga durata prevista nell'art. 79 par. 2 lett. a TFUE non è stata ancora esercitata dall'Unione e dunque resta degli Stati (v. par. 44 della sentenza), i quali possono discrezionalmente decidere di rifiutare il visto, anche senza darne motivazione.

Da un lato, quindi, tale esclusione non permette di fondare sul diritto UE (in particolare sugli artt. 4 e 18 della Carta) l'obbligo di concedere un visto temporaneo a chi, in caso di rifiuto, si trovi in un paese terzo esposto a un sicuro pericolo di persecuzione e trattamenti inumani e degradanti, rimanendo costui costretto ad affrontare "viaggi della speranza" nel Mediterraneo su imbarcazioni di fortuna alla mercé di smugglers senza scrupoli. Un risultato paradossale anche rispetto allo scopo precipuo del codice visti, contenuto nel suo preambolo, di «facilitare i viaggi legittimi e scoraggiare l'immigrazione clandestina».

\section{QUALE ALTRA BASE GIURIDICA NEL DIRITTO INTERNAZIONALE?}

La decisione di rimettere agli Stati piena discrezionalità intorno alla concessione dei visti in circostanze simili a quelle del caso in commento - che avrebbero richiesto all'opposto, secondo l'Avvocato Generale Mengozzi, un obbligo di concessione - in ogni caso, non implica il divieto per gli Stati stessi di concludere intese con sponsor privati per l'apertura di corridoi umanitari, anche se, almeno formalmente, le stesse andranno ripensate rispetto alla base giuridica applicabile, considerato il requisito essenziale dell'espressa volontà del beneficiario di stabilirsi indefinitamente e di permanere sul territorio per chiedere protezionale internazionale.

Sotto questo punto di vista sembra indicativo che non faccia menzione dell'art. 25 del codice visti il più recente protocollo d'intesa siglato in Francia pochi giorni dopo la sentenza della Corte di giustizia, il 13 
marzo 2017, dai Ministeri degli esteri e interni con la Comunità di Sant'Egidio, la Federazione protestante, la Federazione protestante di Mutuo Soccorso, la Conferenza episcopale e la Caritas.

Si ritiene peraltro che una base giuridica sia già rintracciabile nel diritto internazionale, sia generale, sia pattizio. Il riferimento va rivolto, non solo al basilare diritto alla vita, alla libertà e alla sicurezza della propria persona (art. 3 della Dichiarazione Universale) e al diritto di chiedere e godere dell'asilo dalle persecuzioni, riconosciuto dalla all'art. 14.1 della stessa Dichiarazione Universale, ma anche e soprattutto in particolare, al principio di non refoulement (e dunque all'art. 33 della Convenzione di Ginevra) e al divieto di tortura e trattamenti inumani e degradanti contenuto nell'art. 3 della Convenzione europea sui diritti dell'uomo e alla sua efficacia extraterritoriale (su cui ex multis, ad esempio il caso Hirsi), tratta dalla rilevante giurisprudenza della Corte di Strasburgo. A questi poi si aggiungano i diritti dei soggetti più vulnerabili per la loro minore età, e in particolare il principio del superiore interesse dei fanciulli confermato in molti strumenti di diritto internazionale tra cui la citata convenzione di New York, così come il diritto a avere e mantenere una famiglia da cui deriva il diritto al ricongiungimento familiare. Ma soprattutto a questi diritti fanno da sponda gli obblighi per tutti i soggetti di "agire gli uni verso gli altri in spirito di fratellanza" come ricorda chiaramente ancora la Dichiarazione Universale all'art. 1.

La stessa base giuridica costituita da tali principi e regole di diritto internazionale, peraltro, avrebbe potuto giustificare nel caso $X$ e $X$ contro Belgio la necessità della concessione di un VTL «in virtù di obblighi internazionali», a cui l'art. 25 del codice visti appunto rimanda. Anche se la Corte non si è pronunciata sul punto sollevato dal giudice a quo, la discrezionalità degli Stati non dovrebbe essere esercitata senza il rispetto dei limiti posti dagli obblighi derivanti dal diritto internazionale appigliandosi a una interpretazione formalistica della normativa europea. Tale interpretazione risulta troppo fragile rispetto alla necessità di confermare $\mathrm{i}$ «valori umanitari e del rispetto dei diritti dell'uomo sui quali si fonda la costruzione europea... come si afferma rispettivamente negli articoli 2 e 3 del Trattato EU» (conclusioni dell'AG Mengozzi, par. 6).

Inoltre, anche a volere ammettere una diversa lettura più restrittiva di questi obblighi internazionali, in particolare cioè pur sostenendo che il divieto di refoulement possa applicarsi solo nei casi in cui i profughi raggiungano il territorio o i confini degli Stati parte e che per l'ap- 
plicazione degli standard della CEDU i migranti debbano essere sottoposti alla giurisdizione degli Stati membri (e che tale non sia esercitata in una rappresentanza diplomatica all'estero), il rifiuto di concedere un visto per motivi umanitari comporta delle conseguenze per le politiche europee comuni di asilo e avrebbe comunque richiesto l'applicazione dei principi di diritto europeo, Carta dei diritti fondamentali inclusa. Ciò senza considerare che lo stesso codice visti all'art. 32 avrebbe da solo imposto allo Stato belga almeno una motivazione del rifiuto.

\section{QUESTIONI APERTE IN VISTA DELLA DIFFUSIONE DI UNA PRASSI VIRTUOSA IN EUROPA}

Resta la delusione per le parole della Corte che, prendendo le distanze dal lungo e appassionato parere dell'Avvocato Generale espressosi a favore dell'applicazione dell'art. 25 del visa code e della Carta dei diritti fondamentali, in una breve sentenza, ha spazzato via le speranze di quanti attendevano una chiara e coerente presa di posizione dell'Unione a favore della prevalenza dei diritti umani dei migranti in condizione di vulnerabilità e di estremo e urgente bisogno di protezione sulle ottiche stataliste securitarie e protezionistiche.

La Corte sembra avere celato nei passi conclusivi della decisione (paragrafi 48 e 49) le vere ragioni ai suoi occhi (e a quelli degli Stati membri stessi) ostative all'applicazione dell'art. 25 del codice visti e cioè il timore che:

i) le rappresentanze diplomatiche degli Stati membri situate in Stati terzi sarebbero immediatamente diventate il canale preferenziale di richieste di VTL;

ii) si sarebbe in tal modo favorito il fenomeno del c.d. asylum shopping e cioè della scelta dello Stato a cui avanzare richiesta di protezione internazionale direttamente operata dai potenziali beneficiari di protezione.

Secondo molti, e per la stessa pur celata ammissione della Corte, una decisione di segno opposto probabilmente avrebbe fatto definitivamente collassare il già traballante sistema Dublino che gli Stati membri si ostinano a mantenere in vita anche di fronte all'incessante ripetersi dei tragici viaggi della speranza che hanno coinvolto quanti, in condizioni di temere seriamente per la propria vita, non hanno avuto accesso a vie legali alternative, tra cui possono sicuramente comprendersi i "corridoi umanitari" aperti verso l'Italia. 
È certo che la Corte ha perso l'occasione di utilizzare uno strumento già esistente per uniformare una prassi nazionale ad oggi parcellizzata negli Stati membri che la regolano discrezionalmente in modo non univoco. Queste prassi, se ben regolamentate e gestite, all'opposto, permetterebbero l'indubbio vantaggio per lo Stato ospite di controllare (e selezionare) preventivamente gli ingressi sul suo territorio a favore di soggetti che danno maggiori garanzie di integrazione, proprio quando dichiarano espressamente la loro volontà di stabilirsi in Italia e hanno già dei collegamenti con le comunità di accoglienza.

I numeri italiani sono ancora ridotti ma la prassi decennale canadese dimostra le potenzialità del sistema: tra novembre 2015 e luglio 2017 quasi 19.000 siriani hanno beneficiato di sponsorizzazione privata.

Inoltre, sin da ora è evidente l'effetto contagioso della prassi virtuosa (ad oggi hanno seguito l'esempio, la Francia e il Belgio, e arrivano donazioni dall'estero, dalla Germania in primis).

Restano cionondimeno aperte alcune questioni per potere estendere i modelli di intese fin qui adottati: in particolare, la definizione dei criteri di scelta e delle procedure di selezione applicabili in concreto non sembra che possa essere completamente delegata alle ONG-sponsor senza alcun controllo e coordinamento da parte dello Stato, soprattutto in caso di allargamento della prassi a numeri più ampi di beneficiari e nel caso - auspicabile - di coinvolgimento di nuovi sponsor; l'identificazione degli Stati «sicuri», sia di transito sia di accoglienza; l'armonizzazione delle modalità di accoglienza che permetta di offrire e svolgere un percorso di inclusione dei beneficiari secondo un modello di integrazione "adozionale", possibilmente stabilita a diversi livelli, non solo nazionale ma anche europeo. In tale direzione, infatti, si auspica che si diriga anche il recast della normativa europea rilevante tuttora in via di (difficile) elaborazione.

L'applicazione del modello del paternalismo libertario (c.d. nudging, il cui teorizzatore - Richard Thaler - è stato premiato l'anno scorso con il nobel per l'economia o meglio per la behaviuoral economy) potrebbe promuovere queste pratiche virtuose anche in Italia o in altri Stati europei, senza renderle obbligatorie, premiando direttamente o indirettamente quanti decidessero di diventare sponsor. Così avviene già da decenni in Canada, Stato che come si è già ricordato si è fatto sostenitore dell'iniziativa in collaborazione con i privati.

La convinzione che ne è alla base è che, in ottica umanitaria, le rappresentanze consolari all'estero sono molte e quindi diversi potreb- 
bero essere i punti di accesso e controllo dei requisiti a quanti in condizioni di bisogno chiedono protezione negli Stati di transito o prima accoglienza, senza dovere affrontare pericolosi viaggi della speranza. Inoltre, rispetto ad esigenze di sicurezza e integrazione, questo tipo di modello di intervento pubblico-privato permette alle comunità ospiti di conoscere i beneficiari prima che arrivino, predisponendo un piano di accoglienza mirato e calibrato rispetto alle reali possibilità di integrazione. Quest'ultima costituisce lo scopo che caratterizza precipuamente l'iniziativa italiana finalizzata a segnare un percorso condiviso da migranti e società di stabilimento. 